\title{
The Solution of Binary Nonlinear Operator Equations with Applications
}

\author{
Baomin Qiao \\ Department of Mathematics, Shangqiu Normal College, Shangqiu, China \\ Email: bmqiao@126.com
}

Received June 6, 2013; revised July 6, 2013; accepted July 13, 2013

Copyright (C) 2013 Baomin Qiao. This is an open access article distributed under the Creative Commons Attribution License, which permits unrestricted use, distribution, and reproduction in any medium, provided the original work is properly cited.

\begin{abstract}
In this paper, the existence and uniqueness of solution systems for some binary nonlinear operator equations are discussed by using cone and partial order theory and monotone iteration theory, and the iterative sequences which converge to solution of operator equations and error estimates for iterative sequences are also given. Some corresponding results are improved and generalized. Finally, the applications of our results are given.
\end{abstract}

Keywords: Cone and Partial Order; Solution; Nonlinear Binary Operator; Operator Equations

\section{Introduction}

In recent years, more and more scholars have studied binary operator equations and have obtained many conclusions, such as references [1-3] etc. In this paper, we will discuss solutions for ordinal symmetric contraction operator and obtain some general conclusions; some corresponding results of references [4,5] are improved and generalized. Finally, we apply our conclusions to two point boundary value problems with two degree superlinear ordinary differential equations.

In the following, let $E$ always be a real Banach space which is partially ordered by a cone $P, P$ be normal cone of $E, N$ is normal constant of $P$, partial order $\leq$ is determined by $P, \theta$ denotes zero element of $E$. For $u, v \in E$ and $u<v$, let

$$
D=[u, v]=\{x \in E: u \leq x \leq v\}
$$

denotes an ordering interval of $E$.

The concepts of normal cone and partially order, mixed monotone operator, coupled solutions of operator equations etc. see [6].

Definition 1.1. Let $A: D \times D \rightarrow E$ be binary operator, $A$ is said to be $L$-ordering symmetric contraction operator if there exists a bounded linear operator $L: E \rightarrow E$, which its spectral radius $r(L)<1$ such that

$$
A(y, x)-A(x, y) \leq L(y-x)
$$

for any $x, y \in D, x \leq y$, where $L$ is called contraction operator of $A$.

\section{Main Results}

Theorem 2.1. Let $A: D \times D \rightarrow E$ be $L$-ordering symmetric contraction operator, and there exists a $\alpha \in[0,1)$, for any $u \leq x_{1} \leq x_{2} \leq v, u \leq y_{2} \leq y_{1} \leq v$ such that

$$
A\left(x_{2}, y_{2}\right)-A\left(x_{1}, y_{1}\right) \geq-\alpha\left(x_{2}-x_{1}\right) \text {. }
$$

If condition

$$
u \leq A(u, v), A(v, u) \leq v-\alpha(v-u)
$$

or

$\left(\mathrm{H}_{2}\right) \quad u+\alpha(v-u) \leq A(u, v), A(v, u) \leq v$

holds, then the following statements hold:

$\left(C_{1}\right) \quad A(x, x)=x$ has a unique solution $x^{*} \in D$, and for any coupled solution $x, y \in D$ such that $x=y=x^{*}$;

$\left(\mathrm{C}_{2}\right)$ For any $x_{0}, y_{0} \in D$, we make up symmetric iterative sequences

$$
\begin{aligned}
& x_{n}=(\alpha+1)^{-1}\left(A\left(x_{n-1}, y_{n-1}\right)+\alpha x_{n-1}\right), \\
& y_{n}=(\alpha+1)^{-1}\left(A\left(y_{n-1}, x_{n-1}\right)+\alpha y_{n-1}\right), \\
& n=1,2,3, \cdots,
\end{aligned}
$$

then

$$
x_{n} \rightarrow x^{*}, y_{n} \rightarrow x^{*}(n \rightarrow \infty),
$$

and for any $\beta \in(r(L), 1)$, there exists a natural numbers $m$, if $n \geq m$, we get error estimates for iterative sequences (2):

$$
\left\|x_{n}\left(y_{n}\right)-x^{*}\right\| \leq 2 N\left(\frac{\alpha+\beta}{\alpha+1}\right)^{n}\|u-v\| .
$$




\section{Proof. Set}

$$
B(x, y)=\frac{1}{\alpha+1}[A(x, y)+\alpha x],
$$

if condition $\left(\mathrm{H}_{1}\right)$ or $\left(\mathrm{H}_{2}\right)$ holds, then it is obvious

$$
u \leq B(u, v), B(v, u) \leq v,
$$

by (1), we easily prove that $B: D \times D \rightarrow E$ is mixed monotone operator, and for any $u \leq x \leq y \leq v$ such that

$$
\theta \leq B(y, x)-B(x, y) \leq H(y-x),
$$

where

$$
H=(\alpha+1)^{-1}(L+\alpha I)
$$

is a bounded linear operator, $I$ is identical operator.

By the mathematical induction, we easily prove that

$$
\theta \leq B^{n}(y, x)-B^{n}(x, y) \leq H^{n}(y-x) \quad u \leq x \leq y \leq v,
$$

where

$$
B^{n}(x, y)=B\left(B^{n-1}(x, y), B^{n-1}(y, x)\right), x, y \in D, n \geq 2 .
$$

By the character of normal cone $P$, we implies

$$
\left\|B^{n}(y, x)-B^{n}(x, y)\right\| \leq N\left\|H^{n}\right\|\|x-y\|, u \leq x \leq y \leq v .
$$

For any $\beta \in(r(L), 1)$, since

$$
\lim _{n \rightarrow \infty}\left\|H^{n}\right\|^{\frac{1}{n}}=r(H) \leq \frac{\alpha+r(L)}{\alpha+1}<\frac{\alpha+\beta}{\alpha+1}<1,
$$

so there exists a natural numbers $m$, if $n \geq m$, such that

$$
\left\|H^{n}\right\|<\left(\frac{\alpha+\beta}{\alpha+1}\right)^{n}
$$

and constant $N\left\|H^{m}\right\|<1$.

Considering mixed monotone operator $B^{m}$ and constant $N\left\|H^{m}\right\|$, by Theorem 3 in reference [3], then we know $B^{m}(x, x)=x$ has an unique solution $x^{*}$, and for any coupled solution $x, y \in D$ such that

$$
x=y=x^{*} .
$$

From

$$
\begin{aligned}
& B^{m}\left(B\left(x^{*}, x^{*}\right), B\left(x^{*}, x^{*}\right)\right) \\
& =B\left(B^{m}\left(x^{*}, x^{*}\right), B^{m}\left(x^{*}, x^{*}\right)\right)=B\left(x^{*}, x^{*}\right)
\end{aligned}
$$

and uniqueness of solutions with $B^{m}(x, x)=x$, then we have $B\left(x^{*}, x^{*}\right)=x^{*}$ and $A\left(x^{*}, x^{*}\right)=x^{*}$.

We take note of that $A(x, x)=x$ and $B(x, x)=x$ have same coupled solution, therefore coupled solution for $B(x, x)=x$ must be coupled solution for $B^{m}(x, x)=$ $x$, consequently, $\left(\mathrm{C}_{1}\right)$ has been proved.

Considering that iterative sequence (2) and set iterative sequences:

$$
u_{n}=B\left(u_{n-1}, v_{n-1}\right), v_{n}=B\left(v_{n-1}, u_{n-1}\right),
$$

where $u_{0}=u, v_{0}=v$, it is obvious that

$$
\begin{aligned}
& x_{n}=B\left(x_{n-1}, y_{n-1}\right), y_{n}=B\left(y_{n-1}, x_{n-1}\right), \\
& \theta \leq v_{n}-u_{n} \leq H^{n}(v-u),
\end{aligned}
$$

by the mathematical induction and character of mixed monotone of $B$, then

$$
u_{n} \leq x^{*} \leq v_{n}, u_{n} \leq x_{n} \leq v_{n}, u_{n} \leq y_{n} \leq v_{n},
$$

hence

$$
\begin{aligned}
& \left\|x_{n}\left(y_{n}\right)-u_{n}\right\| \leq N\left\|v_{n}-u_{n}\right\|, \\
& \left\|x^{*}-u_{n}\right\| \leq N\left\|v_{n}-u_{n}\right\|, n=1,2,3, \cdots,
\end{aligned}
$$

moreover, if $n \geq m$, we get

$$
\begin{aligned}
\left\|x_{n}\left(y_{n}\right)-x^{*}\right\| & \leq 2 N\left\|v_{n}-u_{n}\right\| \leq 2 N\left\|H^{n}\right\|\|v-u\| \\
& \leq 2 N\left(\frac{\alpha+\beta}{\alpha+1}\right)^{n}\|u-v\|,
\end{aligned}
$$

consequently, $x_{n} \rightarrow x^{*}, y_{n} \rightarrow x^{*}(n \rightarrow \infty)$.

Remark 1. When $\alpha=0$, Theorem 1 in [4] is a special case of this paper Theorem 2.1 under condition $\left(\mathrm{H}_{1}\right)$ or $\left(\mathrm{H}_{2}\right)$.

Corollary 2.1. Let $A: D \times D \rightarrow E$ be $L$-ordering symmetric contraction operator, if there exists a $\alpha \in[0,1)$ such that $A$ satisfies condition of Theorem 2.1, then $\left(C_{1}\right)$, $\left(\mathrm{C}_{2}\right)$ hold and the following statements holds:

$\left(\mathrm{C}_{3}\right)$ For any $\beta \in(r(L), 1)$ and $\alpha+\beta<1$, we make up iterative sequences

$$
\begin{aligned}
& u_{n}=A\left(u_{n-1}, v_{n-1}\right), \\
& v_{n}=A\left(v_{n-1}, u_{n-1}\right)+\alpha\left(v_{n-1}-u_{n-1}\right), \\
& n=1,2,3, \cdots,
\end{aligned}
$$

or

$$
\begin{aligned}
& u_{n}=A\left(u_{n-1}, v_{n-1}\right)-\alpha\left(v_{n-1}-u_{n-1}\right), \\
& v_{n}=A\left(v_{n-1}, u_{n-1}\right), \\
& n=1,2,3, \cdots,
\end{aligned}
$$

where $u_{0}=u, v_{0}=v$ thus $u_{n} \rightarrow x^{*}, v_{n} \rightarrow x^{*}(n \rightarrow \infty)$, and there exists a natural numbers $m$, if $n \geq m$, we have error estimates for iterative sequences (3) or (4):

$$
\left\|u_{n}\left(v_{n}\right)-x^{*}\right\| \leq N(\alpha+\beta)^{n}\|u-v\| .
$$

Proof. By the character of mixed monotone of $A$, then (1) and $\left(\mathrm{C}_{1}\right),\left(\mathrm{C}_{2}\right)$ [in $(1),\left(\mathrm{C}_{2}\right)$ where $\alpha=0$ ] hold. In the following, we will prove $\left(\mathrm{C}_{3}\right)$.

Consider iterative sequence (3), since

$$
u \leq x^{*} \leq v,
$$

so we get

$$
\begin{aligned}
u_{1} & =A(u, v) \leq A\left(x^{*}, x^{*}\right) \\
& =x^{*} \leq A(v, u)=v_{1}-\alpha(v-u) \leq v_{1},
\end{aligned}
$$


by the mathematical induction, we easily prove

$$
u_{n} \leq x^{*} \leq v_{n}, n \geq 1
$$

hence

$$
\theta \leq x^{*}-u_{n} \leq v_{n}-u_{n}, \theta \leq v_{n}-x^{*} \leq v_{n}-u_{n} .
$$

It is clear

$$
\begin{aligned}
\theta & \leq v_{n}-u_{n} \leq(L+\alpha I)\left(v_{n-1}-u_{n-1}\right) \\
& =(L+\alpha I)^{n}(v-u), n \geq 1 .
\end{aligned}
$$

For any $\beta \in(r(L), 1), \alpha+\beta<1$, since

$$
\lim _{n \rightarrow \infty}\left\|(L+\alpha I)^{n}\right\|^{\frac{1}{n}}=r(L+\alpha I) \leq r(L)+\alpha<\alpha+\beta<1,
$$

thus there exists a natural numbers $m$, if $n \geq m$, such that

$$
\left\|(L+\alpha I)^{n}\right\|<(\alpha+\beta)^{n} \text {. }
$$

Moreover,

$$
\begin{aligned}
\left\|u_{n}\left(v_{n}\right)-x^{*}\right\| & \leq N\left\|(L+\alpha I)^{n}\right\|\|u-v\| \\
& \leq N(\alpha+\beta)^{n}\|u-v\|,(n \geq m),
\end{aligned}
$$

consequently, $u_{n} \rightarrow x^{*}, \quad v_{n} \rightarrow x^{*},(n \rightarrow \infty)$.

Similarly, we can prove (4).

Theorem 2.2. Let $A: D \times D \rightarrow E$ be $L$-ordering symmetric contraction operator, if there exists a $\alpha \in[0,1)$ such that

$$
(1-\alpha) u \leq A(u, v), A(v, u) \leq(1-\alpha) v,
$$

then the following statements holds:

$\left(\mathrm{C}_{4}\right)$ Operator equation

$$
A(x, x)=(1-\alpha) x
$$

has an unique of solution $x^{*} \in D$, and for its any coupled solution $x, y \in D$, such that $x=y=x^{*}$;

$\left(\mathrm{C}_{5}\right)$ For any $x_{0}, y_{0}, w_{0}, z_{0} \in D$, we make up symmetric iterative sequence

$$
\begin{aligned}
& x_{n}=\frac{1}{1-\alpha} A\left(x_{n-1}, y_{n-1}\right), \\
& y_{n}=\frac{1}{1-\alpha} A\left(y_{n-1}, x_{n-1}\right), \\
& n=1,2,3, \cdots, \\
& w_{n}=A\left(w_{n-1}, z_{n-1}\right)+\alpha w_{n-1}, \\
& z_{n}=A\left(z_{n-1}, w_{n-1}\right)+\alpha z_{n-1}, \\
& n=1,2,3, \cdots,
\end{aligned}
$$

then

$$
\begin{aligned}
& x_{n} \rightarrow x^{*}, y_{n} \rightarrow x^{*}, \\
& w_{n} \rightarrow x^{*}, z_{n} \rightarrow x^{*}(n \rightarrow \infty),
\end{aligned}
$$

and that for any $\beta \in(r(L), 1)$ and $\alpha+\beta<1$, there exists a natural numbers $m$, if $n \geq m$, then we have error estimates for iterative sequences (6) and (7) respectively:

$$
\begin{aligned}
& \left\|x_{n}\left(y_{n}\right)-x^{*}\right\| \leq 2 N\left(\frac{\beta}{1-\alpha}\right)^{n}\|u-v\|, \\
& \left\|w_{n}\left(z_{n}\right)-x^{*}\right\| \leq 2 N(\alpha+\beta)^{n}\|u-v\| .
\end{aligned}
$$

Proof. Set

$$
B(x, y)=\frac{1}{1-\alpha} A(x, y)
$$

or

$$
C(x, y)=A(x, y)+\alpha x,
$$

we can prove this theorem imitate proof of Theorem 2.1, over.

Similarly, we can prove following theorems.

Theorem 2.3. Let $A: D \times D \rightarrow E$ be $L$-ordering symmetric contraction operator, if there exists a $\alpha \in[0,1)$ such that

$$
u+\alpha v \leq A(u, v), A(v, u) \leq v+\alpha u,
$$

then the following statements holds:

$\left(\mathrm{C}_{6}\right)$ Equation

$$
A(x, x)=(1+\alpha) x
$$

has an unique solution $x^{*} \in D$, and for any coupled solution $x, y \in D$ such that $x=y=x^{*}$;

$\left(\mathrm{C}_{7}\right)$ For any $x_{0}, y_{0} \in D$, we make up symmetric iterative sequence

$$
\begin{aligned}
& x_{n}=\frac{1}{\alpha+1} A\left(x_{n-1}, y_{n-1}\right), \\
& y_{n}=\frac{1}{\alpha+1} A\left(y_{n-1}, x_{n-1}\right), \\
& n=1,2,3, \cdots,
\end{aligned}
$$

then that $x_{n} \rightarrow x^{*}, y_{n} \rightarrow x^{*}(n \rightarrow \infty)$, moreover, $\beta \in(r(L), 1)$, there exist natural number $m$, if $n \geq m$, then we have error estimates for iterative sequence (9):

$$
\left\|x_{n}\left(y_{n}\right)-x^{*}\right\| \leq 2 N\left(\frac{\beta}{\alpha+1}\right)^{n}\|u-v\| ;
$$

$\left(\mathrm{C}_{8}\right)$ For any $\beta \in(r(L), 1)(\alpha+\beta<1), w_{0}, z_{0} \in D$, we make up symmetry iterative sequence

$$
\begin{aligned}
& w_{n}=A\left(w_{n-1}, z_{n-1}\right)-\alpha z_{n-1}, \\
& z_{n}=A\left(z_{n-1}, w_{n-1}\right)-\alpha w_{n-1}, n \geq 1 .
\end{aligned}
$$

Then

$$
w_{n} \rightarrow x^{*}, z_{n} \rightarrow x^{*}(n \rightarrow \infty),
$$

and there exists a natural numbers $m$, if $n \geq m$, we have error estimates for iterative sequence (8). 
Remark 2. When $\alpha=0$, Corollary 2 in [4] is a special case of this paper Theorem $2.1-2.3$.

Remark 3. The contraction constant of operator in [5] is expand into the contraction operator of this paper.

Remark 4. Operator $A$ of this paper does not need character of mixed monotone as operator in [6].

\section{Application}

We consider that two point boundary value problems for two degree super linear ordinary differential equations

$$
\begin{gathered}
x^{\prime \prime}+a(t) x^{m}+\frac{1}{1+b(t) x}=0, t \in[0,1], \\
x(0)=x^{\prime}(1)=0 .(m \geq 2) .
\end{gathered}
$$

Let $k(t, s)$ be Green function with boundary value problem (7), that is

$$
k(t, s)=\min \{t, s\}= \begin{cases}t, & t \leq s, \\ s, & s<t .\end{cases}
$$

then that the solution with boundary value problem (7) and solution for nonlinear integral equation with type of Hammerstein

$$
x(t)=\int_{0}^{1} k(t, s)\left\{a(s)[x(s)]^{m}+(1+b(s) x(s))^{-1}\right\} \mathrm{d} s
$$

is equivalent, where

$$
\max _{t \in[0,1]} \int_{0}^{1} k(t, s) \mathrm{d} s=\frac{1}{2} .
$$

Theorem 3.1. Let $a(t), b(t)$ are nonnegative continuous function in $[0,1]$

$$
p=\max _{t \in[0,1]} a(t), q=\max _{t \in[0,1]} b(t) .
$$

If $p<1, m p+q<2$, then boundary value problem (7) have an unique solution $x^{*}(t)$ such that

$$
0 \leq x^{*}(t) \leq 1 \quad t \in[0,1] ;
$$

Moreover, for any initial function $x_{0}(t), y_{0}(t)$ such that

$$
0 \leq x_{0}(t) \leq 1,0 \leq y_{0}(t) \leq 1 \quad(t \in[0,1]),
$$

we make up iterative sequence

$$
\begin{aligned}
& x_{n}(t)=\int_{0}^{1} k(t, s)\left\{a(s)\left[x_{n-1}(s)\right]^{n}+\frac{1}{1+b(s) y_{n-1}(s)}\right\} \mathrm{d} s, \\
& y_{n}(t)=\int_{0}^{1} k(t, s)\left\{a(s)\left[y_{n-1}(s)\right]^{m}+\frac{1}{1+b(s) x_{n-1}(s)}\right\} \mathrm{d} s, \\
& n=1,2,3, \cdots .
\end{aligned}
$$

Then $x_{n}(t), y_{n}(t)$ uniform convergence to $x^{*}(t)$ on $[0,1]$, and we have error estimates

$$
\begin{aligned}
& \left|x_{n}(t)\left(y_{n}(t)\right)-x^{*}(t)\right| \leq 2\left(\frac{m p+q}{2}\right)^{n}, \\
& t \in[0,1], n=1,2,3, \cdots .
\end{aligned}
$$

Proof. Let

$$
E=C[0,1], P=\{x \in E \mid x(t) \geq 0, t \in[0,1]\},
$$

$\|x\|=\max _{t \in[0,1]}|x(t)|$ denote norm of $E$, then that $E$ has become Banach space, $P$ is normal cone of $E$ and its normal constant $N=1$. It is obvious that integral Equation (8) is transformed to operator equation $A(x, x)=x$, where

$$
A(x, y)(t)=\int_{0}^{1} k(t, s)\left\{a(s)[x(s)]^{m}+\frac{1}{1+b(s) y(s)}\right\} \mathrm{d} s,
$$

$t \in[0,1]$.

Set

$$
u=u(t) \equiv 0, v=v(t) \equiv 1,
$$

then $D=[0,1]$ denote ordering interval of $E$, $A: D \times D \rightarrow E$ is mixed monotone operator , and

$$
0 \leq A(0,1), A(1,0) \leq \frac{1+p}{2}<1 .
$$

Set

$$
L x(t)=\int_{0}^{1} k(s, t)[m a(s)+b(s)] x(s) \mathrm{d} s, t \in[0,1],
$$

then $L: E \rightarrow E$ is bounded linear operator, its spectral radius

$$
r(L) \leq \frac{m p+q}{2}<1,
$$

and for any $x, y \in E, \quad 0 \leq x(t) \leq y(t) \leq 1$ such that

$$
0 \leq A(y, x)(t)-A(x, y)(t) \leq L(y-x)(t),
$$

that is, $A$ is $L$-ordering symmetric contraction operator, by Theorem 2.1 (where $\alpha=0$ ), then Theorem 3.1 has be proved.

\section{Acknowledgements}

Supported by the Natural Science Foundation of Henan under Grant 122300410425; the NSF of Henan Education Bureau (2000110019); Supported by the NSF of Shangqiu (200211125).

\section{REFERENCES}

[1] D.-J. Guo and V. Lakshmikantham, "Coupled Fixed Points of Nonlinear Operators with Applications," Nonlinear Analysis, Vol. 11, No. 5, 1987, pp. 623-632. doi:10.1016/0362-546X(87)90077-0

[2] Y. Sun, "A Fixed Point Theorem for Mixed Monotone 
Operators with Applications," Journal of Mathematical Analysis and Applications, Vol. 21, No. 6, 1991, pp. 240-252.

[3] Q.-Z. Zhang, "Contraction Mapping Principle of Mixed Monotone Mapping and Applications," Henan Science, Vol. 18, No. 2, 2000, pp. 121-125.

[4] J.-X. Sun and L.-H. Liu, "Iterative Solutions for Nonlinear Operator Equation with Applications," Acta Mathe- matica Scientia, Vol. 13, No. 3, 1993, pp. 141-145.

[5] Q.-Z. Zhang, "Iterative Solutions of Ordering Symmetric Contraction Operator with Applications," Journal of Engineering Mathematics, Vol. 17, No. 2, 2000, pp. 131134.

[6] X.-L. Yan, "The Fixed Point Theorems for Mixed Monotone Operator with Application," Mathematical Application, Vol. 4, No. 4, 1991, pp. 107-114. 